\title{
Impacto del Ecodriving sobre las emisiones y consumo de combustible en una ruta de Quito
}

\author{
(Impact of Ecodriving on fuel emissions \\ and consumption on road of Quito)
}

\author{
Julio Leguísamo Milla ${ }^{1}$, Edilberto Llanes Cedeño ${ }^{1}$, Juan Rocha Hoyos ${ }^{1}$
}

\begin{abstract}
Resumen
Este artículo presenta los resultados del impacto del ecodriving en el consumo de combustible y los factores de emisión de gases contaminantes de un vehículo a gasolina al efectuar una prueba en ruta, en la ciudad de Quito ubicada a 2810 msnm. Se seleccionó una ruta con tráfico validada por el Centro de Transferencia Tecnológica para la Capacitación e Investigación de Control de Emisiones Vehiculares (CCICEV). Como vehículo de pruebas se utilizó un Aveo Family por ser el de mayor venta en la ciudad. Para la medición del consumo de combustible y la concentración de emisiones los equipos utilizados fueron un analizador de gases onboard y un canister. Se determinó la fiabilidad de los datos y el éxito de la experimentación mediante el software STATGRAPHICS Centurion XVI. Los resultados muestran diferencias significativas en el consumo de combustible y las emisiones contaminantes de CO y NOx al aplicar una conducción eficiente, a excepción de los HC en los cuales no hay una diferencia significativa, pero la emisión es menor en conducción normal para este caso.
\end{abstract}

\section{Palabras clave}

Conducción ecológica; altura; factores de emisión; prueba en ruta; vehículo.

\begin{abstract}
This article presents the results of the impact of ecodriving on fuel consumption and the emission factors of polluting gases from a gasoline vehicle when performing road testing in the city of Quito, located at 2810 meters above sea level. A route with traffic validated by the Technology Transfer Center has been selected for the training and investigation of vehicle emission control. A Chevrolet Aveo Family was used as a test vehicle "considering is the bestselling vehicle in the city at the moment. And the equipment used to measure fuel consumption and the concentration of emissions was an onboard gas analyzer and a canister. The success and the measuring of the data in this experimentation were determined using the STATGRAPHICS Centurion XVI software. The results showed significant differences in fuel consumption and pollutant emissions of CO and NOx when applying an efficient driving, except for the HC in which there is no significant difference, but the emission is lower when is used on standard driving.
\end{abstract}

\section{Keywords}

Ecodrive; Height; emission factor; route test; vehicle.

\section{Introducción}

Los gases que emiten los autos son una fuente de contaminantes atmosféricos y gases de efecto invernadero. Su importancia es cada vez mayor, por sus consecuencias en el calentamiento global, consumo de energía y salud mundial (Andrade-Castañeda, Arteaga-Céspedes y Segura-Madrigal, 2017). El sector del transporte es uno de los más grandes emisores de Gases de efecto invernadero (GEI) a pesar de los avances en el campo de la tecnología en los vehículos y las mejoras en la calidad de combustible (Emberger, 2017). Este sector representó el 25.6 \% del consumo mundial de energía en 2015. Se espera que aumente un $1.1 \%$ cada año hasta 2040

1 Universidad Internacional SEK, Quito-Ecuador (julio.leguisamo@uisek.edu.ec, carlos.rocha@uisek.edu.ec, antonio.llanes@ uisek.edu.ec) 
(Energy Information Administration, 2017). Hay dos tipos de medidas políticas para reducir las emisiones y el consumo de combustible; las tecnológicas: desarrolladas por los fabricantes de vehículos, y operativas (Bottiglione, Contursi, Gentile y Mantriota, 2014). Las medidas operativas son la aplicación de medidas específicas de gestión del tráfico, por ejemplo, límites de velocidad dinámicos, la optimización en la coordinación de señales de tráfico, rampa de medición y medidas para influir en el comportamiento del conductor o programas diseñados para cambiar el estilo de conducción conocidos como ecodriving, ecodrive, conducción eficiente o conducción ecológica (Betella y Verschure, 2016).

La conducción ecológica tiene como objetivo cambiar el comportamiento del conductor a través de un consejo simple como mantener una velocidad constante, acelerar moderadamente, anticipar el flujo de tráfico y aprovechar la inercia del vehículo (Baric, Zovak y Perisa, 2013). El consejo está diseñado para reducir el consumo de combustible y, por lo tanto, reducir las emisiones de gases de efecto invernadero, pero además se han evidenciado mejoras en la seguridad del tráfico y el flujo de tráfico (Yanzhi y Hanyan, Haobing, Liu, Rodgers y Guensler, 2017). El ecodriving en muchos países del primer mundo es bien conocido como una estrategia viable para disminuir el consumo de combustible y las emisiones (Andrieu y Pierre, 2014). Se han evaluado los beneficios del ecodriving a través de la aplicación en el mundo real, a través de los datos de actividad de vehículos simulados, o mediante una combinación de ambos. En las implementaciones del mundo real, los ahorros de combustible observados varían desde $2 \%$ a 50 \% y la reducción de emisiones contaminantes varía de 2 \% a 40 \% (Magaña, 2014; A y Yasunori, 2013; O. Orfila et al., 2017; Morello et al., 2016; Fernández-Rodríguez, Fernández-Cardador y Cucala, 2015; Stillwater, Kurani yMokhtarian, 2017).

Se debe considerar que la altura influye directamente en la combustión debido a que se reduce la cantidad de oxígeno en la mezcla, y bajo estas condiciones es menos eficiente y se emite más contaminantes (Leguisamo, Celi, Noroña y Puente, 2018; Rocha-Hoyos, Tipanluisa, Zambrano y Portilla, 2018). En ciudades de altura como QuitoEcuador, la combustión se realiza con un $27 \%$ menos oxígeno que a nivel del mar, en lo que respecta a su geografía al encontrarse rodeada de montañas se dificulta la circulación de los gases de escape y por estar asentada en la mitad del mundo existe una mayor insolación lo que produce una alta formación de contaminantes fotoquímicos (Secretaría de Ambiente, 2017). En esta ciudad los vehículos son los que más contaminan el aire, el 52 \% de emisiones contaminantes en la capital provienen de esa fuente, el parque automotor que en los últimos 10 años ha tenido variaciones anuales que oscilan entre el $5 \%$ y $10 \%$, lo que ha significado la incorporación entre 15000 y 35000 vehículos por año, la tendencia permite estimar que para el año 2030 se tendría un parque de aproximadamente de 1150000 vehículos (Movilidad, 2014).

Según el estudio realizado por Rodríguez, Virguez, Rodríguez y Behrentz (2016) en la ciudad de Bogotá se determinó que al aplicar ecodriving las emisiones anuales se reducirían en un $12 \%$ para $\mathrm{CO}_{2}, 13 \%$ para $\mathrm{CO}$ y HC, y $24 \%$ para $\mathrm{NO}_{x^{\prime}}$ y además se lograba un ahorro en el consumo de combustible de 35 y 85 millones de galones por año. En Pereira, ciudad colombiana, ubicada a 1441 msnm, en una región montañosa, se desarrolló un estudio de conducción eficiente donde se obtuvo un aumento en el rendimiento del $15 \%$ y $20 \%$ en pruebas de laboratorio y ruta, respectivamente (Castillo, Restrepo, Tibaquirá y Quirama, 2019).

La presente investigación se realiza en la ciudad de Quito-Ecuador, la que se encuentra localizada a una altura media de 2810 msnm, el objetivo del estudio es evaluar el consumo de 
combustible y las emisiones contaminantes ( $\mathrm{CO}, \mathrm{HC}$ y $\mathrm{NO}_{\mathrm{x}}$ ) mediante prueba en ruta para la comprobación del ecodriving como estrategia de manejo y mejora en la eficiencia de un vehículo.

\section{Metodología}

En este trabajo de investigación se adoptó una estrategia para evaluar el consumo de combustible y los factores de emisión de un vehículo al realizar una prueba en ruta en la ciudad de Quito. Determinando dos variables de estudio, la primera consiste en una conducción normal y la segunda está determinada por una conducción ecodriving. Se conoce como conducción normal del vehículo a la manera de conducir habitualmente durante un recorrido, mientras que el ecodriving consiste en una serie de normas que debe aplicar el conductor al conducir el mismo recorrido. En las pruebas ejecutadas el conductor es el mismo, y primero se ejecutan las pruebas de conducción normal y después de una capacitación de 8 horas se realizan las pruebas de ecodriving.

\subsection{Materiales}

El ecodriving es un conjunto de pasos, técnicas y comportamientos que los conductores emplean para obtener ahorros en términos de uso de combustible, costo del viaje, las emisiones de CO y otros tipos de contaminación provenientes del uso del vehículo (Yanzhi, et al, 2017). Los consejos para conducir con ecodriving en vehículos a gasolina y con transmisión manual son: los cambios de marcha debe hacerse tan pronto como sea posible por lo general entre 2000 y 2500 rpm, circular en marchas largas y a bajas revoluciones, usar la primera marcha solo para poner en movimiento el vehículo, mantener la velocidad de marcha, evitar acelerones y frenazos, decelerar con el freno motor, detener el auto utilizando solo el freno, no utilizar el punto muerto cuando se frena, aprovechar la inercia del vehículo siempre que sea posible, en las paradas no escalar todas las marchas sino mantener la marcha puesta hasta pisar el embrague y detenerse (Beusen, Broekx, Denys, Beckx, Degraeuwe, Gijsbers y Panis, 2009; Ho, Wong y Chang, 2015; Rionda, Martínez, Pañeda, Arbesú y Jiménez, 2012)

Para medir las concentraciones de los gases de escape se utilizó un analizador de gases ONBOARD para determinar las concentraciones de gases de $\mathrm{NO}, \mathrm{HC}, \mathrm{CO}$ y $\mathrm{CO}_{2}$, marca GLOBAL AXION R/S TM, las especificaciones técnicas se detallan en la tabla 1 y el equipo se puede observar en la figura 1.

Tabla 1. Especificación Técnica analizador de gases ONBOARD

\begin{tabular}{|c|c|}
\hline Detalle & Valor \\
\hline Principio de medición $\left(\mathrm{HC}, \mathrm{CO}, \mathrm{CO}_{2}\right)$ & Espectrometría de infrarrojo NDIR \\
\hline Principio de medición $\left(\mathrm{O}_{2}, \mathrm{NO}_{x}\right)$ & Detección electroquímica \\
\hline Tensión de Alimentación & $12-24 \mathrm{~V} \mathrm{DC} / 5-8 \mathrm{~A}$ \\
\hline Indice de Flujo & $5 \mathrm{l} / \mathrm{min}$ \\
\hline $\mathrm{O}_{2}$ Rango de medición/Precisión/Resolución & 0.00-25.00 \% / +- $0.1 \%$ abs. Or +/3 \% rel/0.01 Vol. \% \\
\hline CO Rango de medición/Precisión/Resolución & $0.00-10.00 \% /+-0.02 \%$ abs. Or +/3 \% rel/0.01 Vol. \% \\
\hline NOx Rango de medición/Precisión/Resolución & 0-4000 (ppm)/ +- 25ppm abs. Or +/4 \% rel/1ppm \\
\hline $\mathrm{CO}_{2}$ Rango de medición/Precisión/Resolución & $0.00-16.00 \% /+-0.3 \%$ abs. Or +/3 \% rel/0.01 Vol. \% \\
\hline HC Rango de medición/Precisión/Resolución & $0-4000$ ppm / +- 8 ppm abs. Or +/3 \% rel/ 1 ppm \\
\hline
\end{tabular}


Figura 1. Analizador de gases GLOBAL AXION R/S TM

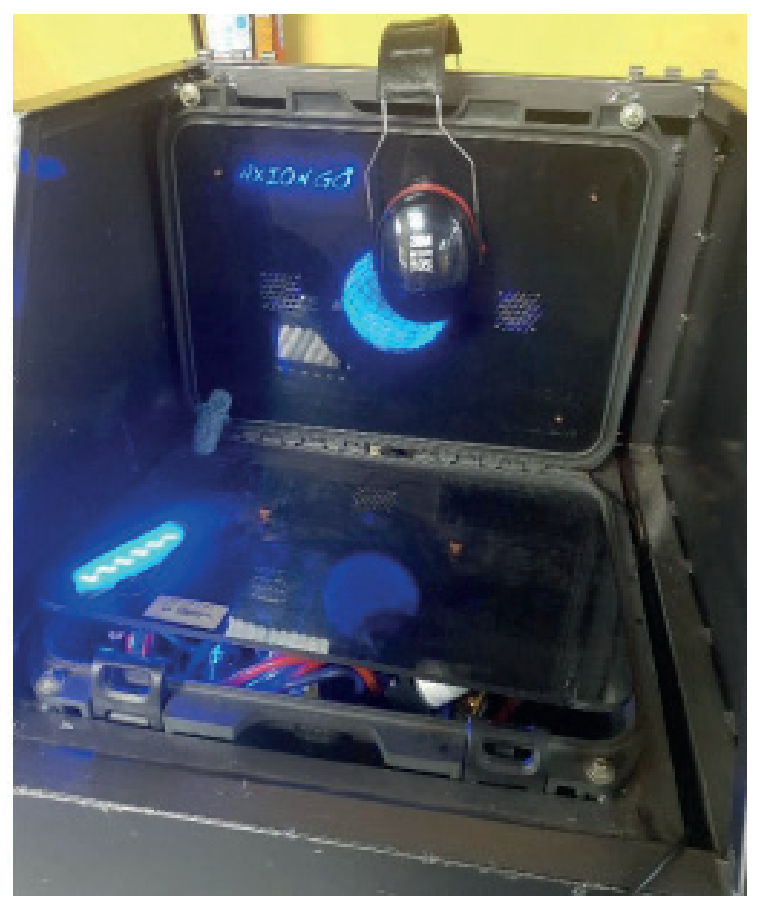

Para las investigaciones experimentales se utilizó el vehículo Chevrolet Aveo Family a gasolina, cuyas características técnicas se detallan en la tabla 2. Se seleccionó este auto, puesto que según las estadísticas de ventas anuales de vehículos en el Ecuador, en la provincia de Pichincha, capital Quito, desde el año 2009 hasta el año 2018 se han vendido 24395 unidades (AEADE, 2018) y es el más vendido en el país. Antes de realizar las pruebas el vehículo fue sometido a una revisión mecánica y electrónica de su estado, se le efectuó un mantenimiento preventivo para garantizar el perfecto estado de los sistemas.

Tabla 2. Características del vehículo de prueba

\begin{tabular}{|l|l|}
\hline \multicolumn{1}{|c|}{ Cilindrada } & \multicolumn{1}{c|}{$\mathbf{1 4 9 8 \mathbf { c m } ^ { 3 }}$} \\
\hline Relación de compresión & $9.5: 1$ \\
\hline Diámetro x carrera & $76.5 \mathrm{~mm} \times 81.5 \mathrm{~mm}$ \\
\hline Potencia & $83 / 5600 \mathrm{hp} / \mathrm{rpm}$ \\
\hline Par motor & $127 / 3000 \mathrm{Nm} / \mathrm{rpm}$ \\
\hline Válvulas & 8 SOHC \\
\hline Sistema de inyección de combustible & MPFI \\
\hline Catalizador de gases de escape & Tres vías \\
\hline Sistema de Encendido & Chispa perdida DIS \\
\hline Motor- tracción & Delantera \\
\hline Transmisión & Manual 5 velocidades \\
\hline Tanque de combustible & $45 \mathrm{~L}$ \\
\hline Kilometraje & 36344 km \\
\hline
\end{tabular}


La cantidad de combustible consumida se mide con un tanque de presión de combustible externo también conocido como canister de marca Liqui Moly que se observa en la figura2 con una capacidad de 5 litros, que se puede presurizar hasta 80 psi. Con este elemento se abastece de combustible a presión al vehículo, que se presuriza de acuerdo a la presión del fabricante. La cantidad de combustible consumido se determina por la diferencia de volumen medidos con probetas antes y después de las pruebas (Rocha et al., 2018)

Figura 2. Canister de combustible Liqui Moly

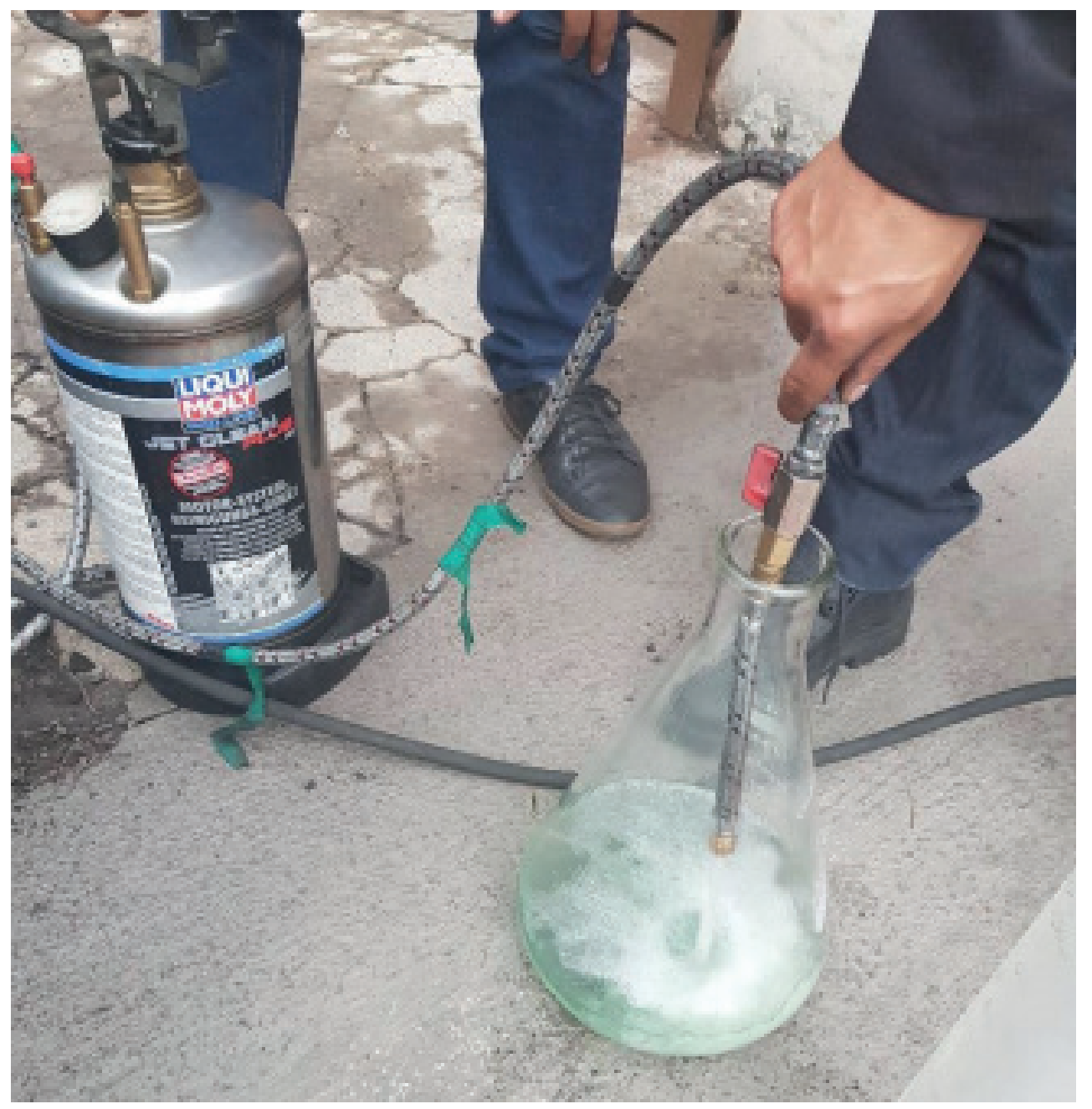

Debido a que el vehículo no viene equipado con tacómetro en el panel de instrumentos, el control de las revoluciones de marcha (2000 a 2500 rpm) se realiza con un escáner automotriz scanner G-Scan2, que cumple la legislación OBDII que prescribe una estandarización de las informaciones de lectura en la línea de datos conforme a las especificaciones de la SAE J 1979, 2002, la conexión del escáner en el vehículo se puede observar en la figura 3. 
Figura 3. Canister de combustible Liqui Moly

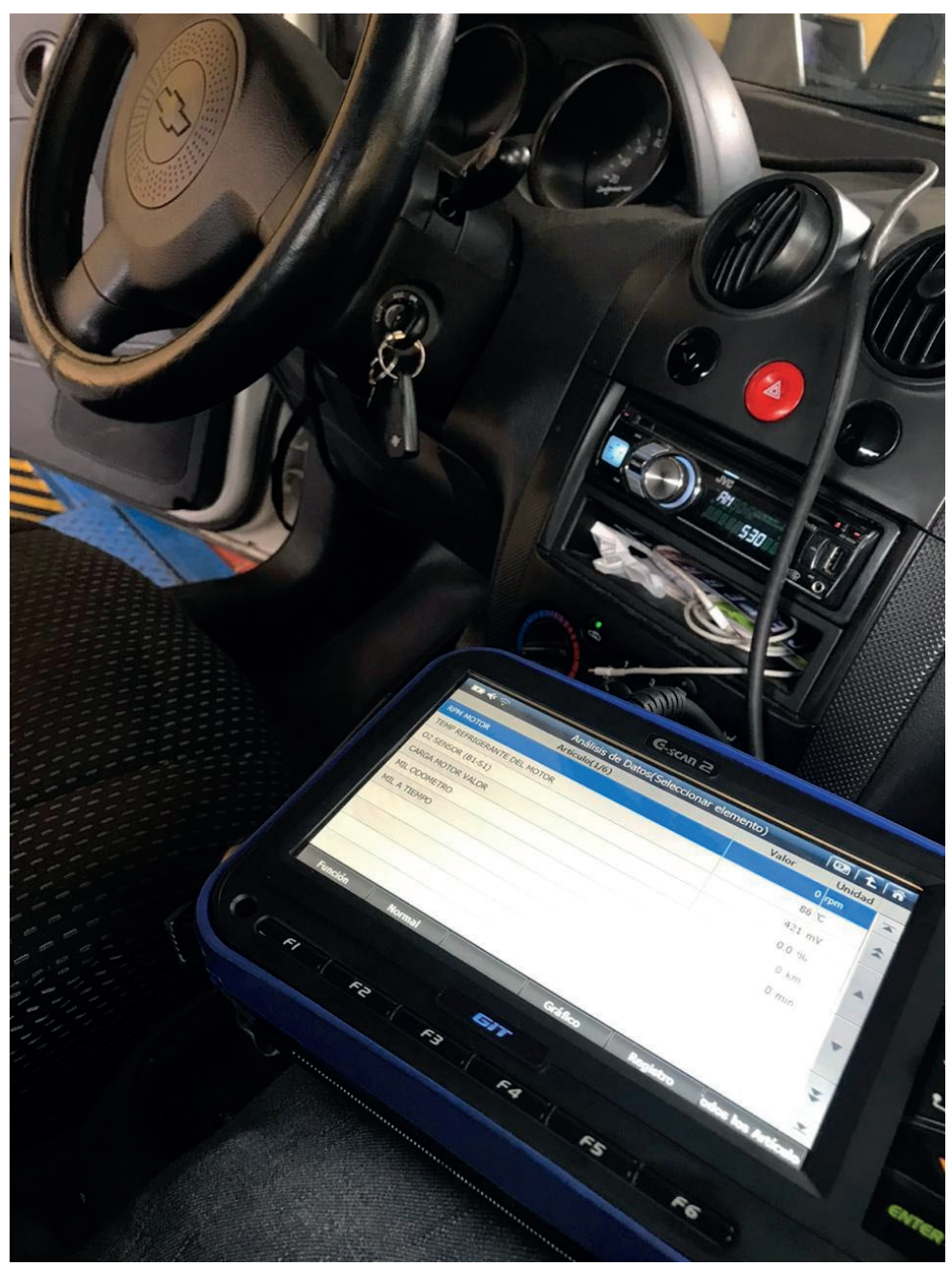

\subsection{Estimación factores de emisión}

\subsubsection{Ruta de prueba}

Para determinar el consumo de combustible y las concentraciones de emisiones contaminantes, se selecciona una ruta desarrollada por el CCICEV de la ciudad de Quito, el cual se ha desarrollado con base en la orografía del terreno, densidad del tráfico e infraestructura vial de la ciudad como se observa en la figura 4. La ruta consta de una distancia de $9990 \mathrm{~m}$ de recorrido suburbano y $8100 \mathrm{~m}$ de recorrido urbano. Tiene una variación de altura de 2399 metros hasta 2870 como punto más alto. La longitud de la ruta se puede distribuir en el $59 \%$ del total de recorrido plano o con pendientes menores 3 grado, el $16 \%$ contiene pendientes de grado 4 , el $6 \%$ pendientes de grado 6 , el $10 \%$ pendientes de grado -4 y el $9 \%$ pendientes de grado -6 (Quichimbla y Solís, 2017). 
Figura 4. Recorrido de la prueba de ruta

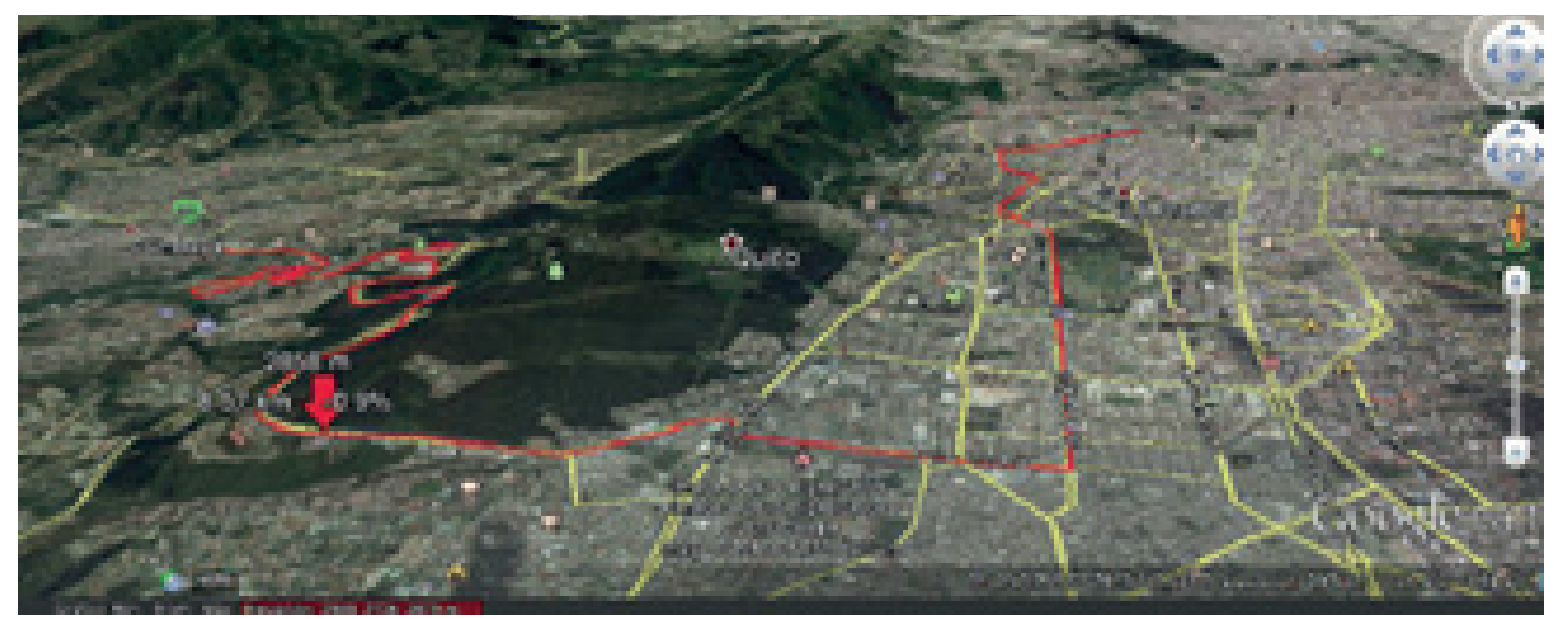

\subsubsection{Protocolo de prueba}

Se desarrolla un protocolo de pruebas que se describe en los siguientes diez puntos: 1) Verificar que el sistema de escape del vehículo no tenga perforaciones ni fugas; 2) Verificar que la presión de los neumáticos sea la recomendada por el fabricante del vehículo; 3) Instalar y asegurar los equipos para la medición de gases ONBOARD, para la medición de consumo de combustible y control de revoluciones; 4) Encender el vehículo y calentar el motor hasta que alcance la temperatura de operación normal que corresponde a $95^{\circ} \mathrm{C}$; 6) Revisar que los accesorios del vehículo estén desactivados; 7) Verificar el correcto funcionamiento de los equipos; 8) Iniciar en la ruta seleccionada; 9) Grabar los datos obtenidos en el computador; y, 10) Repetir el procedimiento para cada prueba con conducción normal y con ecodriving. Se realizaron cinco pruebas de cada tipo de conducción, para determinar si entre los grupos experimentales existe diferencia significativa, se usó el análisis ANOVA con el software STATGRAPHICS Centurion XVI, aplicándose pruebas de comparación múltiple de medias, para este caso la LSD (Least Significant Difference), para un 95 \% de confianza (Guardia et al., 2018).

\subsubsection{Estimación de factores de emisión}

Se establece como base el modelo simplificado de la combustión con el cual se determina las variables dependientes e independientes del experimento. Los valores que se determinan experimentalmente son las concentraciones de volumen expresadas en (\%) de $\mathrm{NO}, \mathrm{HC}, \mathrm{CO}_{\text {y } \mathrm{CO}_{2}} \mathrm{y}$ el consumo de combustible, para el cálculo de los factores de emisión en gramos por kilómetro (g/km).

El análisis dinámico del comportamiento de los gases de escape en los vehículos se expresa en la Ecuación 1, las variables a, b, c, d, e, f y m corresponden a coeficientes estequiométricos desconocidos y determinados por mol de combustible consumido. Se desarrolla un balance de masa respecto a la cantidad de carbono, debido a que como fruto de la combustión se obtiene $\mathrm{CO}_{2}$, $\mathrm{CO}$ y $\mathrm{HC}$ presente en el combustible. El balance de masa para el carbono se puede escribir como la Ecuación 1 (Frey y Eichenberger, 1997; Llanes-Cedeño, Rocha-Hoyos, Peralta-Zurita y Leguísamo-Milla, 2018) 
$\mathrm{CHy}+m\left(0.21 \mathrm{O}_{2}+0.79 \mathrm{~N}_{2}\right) a \mathrm{CO}+b \mathrm{H}_{2} \mathrm{O}+c \mathrm{C}_{3} \mathrm{H}_{6}+d \mathrm{CO}_{2}+e \mathrm{~N}_{2}+f \mathrm{NO}$

Donde: $\mathrm{m}$ = moles de aire consumido por mol de combustible consumido, $\mathrm{a}=$ moles formados de $\mathrm{CO}$ por mol de combustible consumido, $\mathrm{b}=$ moles formados de $\mathrm{H}_{2} \mathrm{O}$ por mol de combustible consumido, $\mathrm{c}=$ moles formados de $\mathrm{C}_{3} \mathrm{H}_{6}$ por mol de combustible consumido, $\mathrm{d}=$ moles formados de $\mathrm{CO}_{2}$ por mol de combustible consumido, $\mathrm{e}=$ moles formadas de $\mathrm{N}_{2}$ por mol de combustible consumido y $f=$ moles formados de NO por mol de combustible consumido.

Al ser el $\mathrm{CO}_{2}$ el gas predominante, en los residuos de combustión se pretende relacionar este gas con el resto de residuos que son indispensables en la relación de este estudio para tal se presenta las Ecuaciones 2, 3, 4 y 5, según Caiza y Portilla (2010):

$1=a+3 c+d$

$a=d^{*} R c o$

$c=d^{*} \mathrm{RHC}$

$d=\frac{1}{R_{C O}+3 R_{H C}+1}$

Donde: $\mathrm{R}_{\mathrm{CO}}$ es la relación de CO respecto a $\mathrm{CO}_{2}$ en porcentaje y $\mathrm{R}_{\mathrm{HC}}$ es la relación de $\mathrm{HC}$ respecto a $\mathrm{CO}_{2}$ en porcentaje.

Se considera como base principal del combustible al octano $\mathrm{C}_{8} \mathrm{H}_{18}$ (equivalente $\mathrm{CH}_{2.25}$ ) y se procede a determinar el peso molecular del combustible como se muestra en la Ecuación 6.

$M W_{C O}=\frac{12 \mathrm{gC}}{\mathrm{molC}} *\left(\frac{1 \mathrm{molC}}{\mathrm{mol} \mathrm{comb}}\right)+\frac{1 \mathrm{gH}}{\mathrm{molH}} *\left(\frac{y^{*} \mathrm{molH}}{\mathrm{mol} \mathrm{comb}}\right)=14,25 \frac{\mathrm{gcomb}}{\mathrm{mol} \mathrm{comb}}=0,01425 \frac{\mathrm{kg} \mathrm{comb}}{\mathrm{mol} \mathrm{comb}}$

Con la densidad del combustible $\left(\mathrm{kg} / \mathrm{m}^{3}\right)$ y consumo por distancia recorrida FC $\left(\mathrm{m}^{3} / \mathrm{km}\right)$, se obtiene el factor de emisión en gramos de cada contaminante por kilómetro recorrido, como se puede observar en las Ecuaciones 7,8 y 9.

$$
\begin{array}{rlrl}
F_{C O} & =\frac{R_{C O}}{R_{C O}+3 R_{H C}+1} & \frac{M W_{C O}}{M W_{c o m b}} \\
F_{H C}=\frac{R_{H C}}{R_{C O}+3 R_{H C}+1} & \frac{M W_{H C}}{M W_{c o m b}} \\
F_{N O}=\frac{R_{N O}}{R_{C O}+3 R_{H C}+1} & \frac{M W_{N O}}{M W_{c o m b}}
\end{array}
$$

Donde $F_{C O}$ corresponde a factor de emisión de CO, $R_{C O}$ razón de \% de CO con respecto a la concentración de $\mathrm{CO}_{2^{\prime}} R_{\mathrm{HC}}$ razón de \% de $\mathrm{HC}$ en cuanto a la concentración de $\mathrm{CO}_{2}, R_{\mathrm{NO}}$ razón de \% de NOx con respecto a la concentración de $\mathrm{CO}_{2}, M W_{C O}$ peso molecular del CO, $M W_{H C}$ peso molecular de $\mathrm{HC}, M W_{N O}$ peso molecular de NO y $M W_{\text {comb }}$ peso molecular del combustible. Como variables independientes se consideran la densidad del combustible $\mathbf{\delta} c o m\left(\mathrm{~kg} / \mathrm{m}^{3}\right)$ con un valor de $748 \mathrm{~kg} / \mathrm{m}^{3}$ a $15^{\circ} \mathrm{C}$ y el consumo de combustible por distancia recorrida en la ruta de prueba. 


\section{Resultados y discusión}

En la tabla 3 se presentan los resultados del consumo de combustible y los factores de emisión en las pruebas, en el análisis de resultados se debe considerar que la altura influye directamente en la combustión debido a la cantidad de oxígeno en la mezcla y bajo estas condiciones, influye en las emisiones y el consumo de combustible. Se consideran 2 escenarios: Escenario 1 (conducción normal) y Escenario 2 (ecodriving).

Tabla 3. Resultados del consumo de combustible y factores de emisión para los modos de conducción

\begin{tabular}{|c|c|c|c|c|c|c|c|c|}
\hline \multirow[b]{2}{*}{ Prueba } & \multicolumn{4}{|c|}{ Conducción Normal (1) } & \multicolumn{4}{|c|}{ Ecodriving (2) } \\
\hline & $\begin{array}{c}\text { Consumo } \\
(1 / \mathrm{km})\end{array}$ & $\begin{array}{c}\mathrm{FCO}(\mathrm{g} / \\
\mathrm{km})\end{array}$ & $\begin{array}{c}\mathrm{FHC}(\mathrm{g} / \\
\mathrm{km})\end{array}$ & $\begin{array}{c}\mathrm{FNO}_{\mathrm{x}}(\mathrm{g} / \\
\mathrm{km})\end{array}$ & $\begin{array}{c}\text { Consumo } \\
(1 / \mathrm{km})\end{array}$ & $\begin{array}{l}\text { FCO } \\
(\mathrm{g} / \mathrm{km})\end{array}$ & $\begin{array}{l}\text { FHC } \\
(\mathrm{g} / \mathrm{km})\end{array}$ & $\begin{array}{l}\mathrm{FNO}_{\mathrm{x}} \\
(\mathrm{g} / \mathrm{km})\end{array}$ \\
\hline 1 & 0.130 & 7.0557 & 0.0916 & 0.9654 & 0.122 & 5.144 & 0.103 & 0.748 \\
\hline 2 & 0.144 & 8.2833 & 0.1271 & 1.0716 & 0.114 & 5.697 & 0.130 & 0.730 \\
\hline 3 & 0.133 & 7.3414 & 0.0960 & 1.0625 & 0.116 & 5.138 & 0.162 & 0.896 \\
\hline 4 & 0.145 & 8.5783 & 0.1598 & 1.0656 & 0.117 & 5.422 & 0.174 & 0.911 \\
\hline 5 & 0.148 & 7.0564 & 0.1333 & 1.0284 & 0.117 & 5.100 & 0.121 & 0.851 \\
\hline Promedio & 0.139 & 7.9830 & 0.1216 & 1.0387 & 0.117 & 5.300 & 0.138 & 0.827 \\
\hline
\end{tabular}

\subsection{Consumo de combustible}

Al aplicar el procedimiento de diferencia mínima significativa conocido como Test LSD de Fisher, con un nivel de confianza del $95 \%$ al consumo de combustible, se determina que existe una diferencia significativa entre los dos modos de conducción, que se indica por la desalineación en la columna de los grupos homogéneos, obteniéndose el mejor resultado con el Ecodriving, como se muestra en la tabla 4.

Tabla 4. Comparación de medias por Fisher LSD del consumo de combustible

\begin{tabular}{|l|l|l|l|}
\hline & \multicolumn{1}{|c|}{ Casos } & \multicolumn{1}{|c|}{ Media } & Grupos Homogéneos \\
\hline Ecodrive & 5 & 0.1172 & $X$ \\
\hline Normal & 5 & 0.14 & $X$ \\
\hline
\end{tabular}

En la figura 5 se representa la comparación de medias, mediante el cual se muestra el impacto del ecodriving en el consumo de combustible, el cual es $0.117 \mathrm{~L} / \mathrm{km}$, mientras que al aplicar conducción normal el valor es 0.139 L/km lo que indica una reducción del 15.82 \% del consumo. Estos márgenes son también el tema de varios estudios con un aproximado de influencia estimado entre 2 y $26 \%$ de ahorro de consumo de combustible, por estudios llevados a cabo en varios países del mundo en diferentes condiciones de altura con respecto al nivel del mar (Larue, Malik, Rakotonirainy y Demmel, 2014; Rutty, Matthews, Andrey y Del Matto, 2013; Ho, Wong y Chang, 2015; Rolim, Baptista, Duarte, Farias yShiftan, 2014; Andrieu y Pierre, 2014; Rodríguez et al., 2016; Castillo et al., 2019). 
Figura 5. Medias comparativas del consumo de combustible según modo de conducción

\section{Medias y $95,0 \%$ de Fisher LSD}

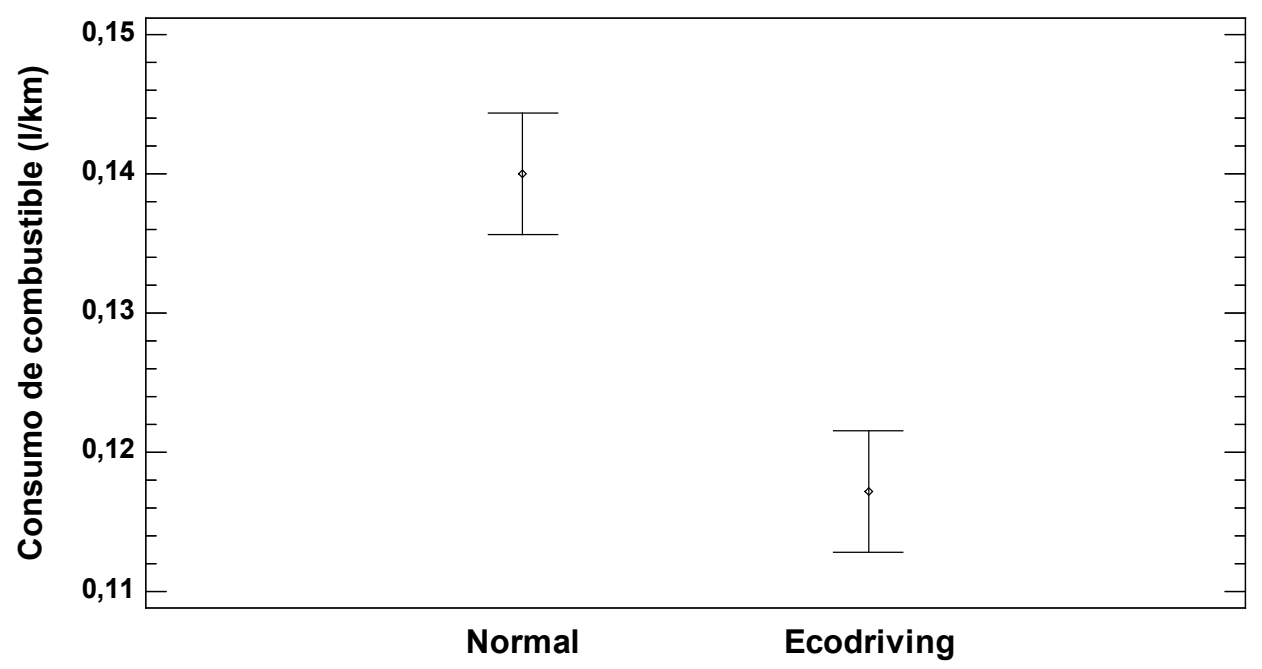

Se obtiene, además, que el consumo de combustible al aplicar el modo de ecodriving en un galón de combustible se puede recorrer 32,104 km mientras que con conducción normal $25.352 \mathrm{~km}$, lo que indica un 26.63 \% de aumento en la autonomía de un galón de combustible, teniendo un valor similar con el estudio de Baric et al. (2013) donde la autonomía de un galón de combustible aumento en 24.38 \% y con respecto al estudio de Castillo et al. (2019) a 1441 msnm en una región montañosa, donde se obtuvo un aumento del 20 \%.

\subsection{Factores de emisión}

Los resultados referentes al factor de emisión de CO se observa en la Tabla 5, en la cual se aprecia que existe diferencia significativa entre los modos de conducción, siendo el modo de ecodriving el de mejor comportamiento con una reducción del $30.84 \%$ de emisiones de Co con relación a la conducción normal.

Tabla 5. Comparativa del factor de emisión de CO

\begin{tabular}{|l|l|l|l|}
\hline & \multicolumn{1}{|c|}{ Casos } & \multicolumn{1}{c|}{ Media } & \multicolumn{1}{c|}{ Grupos Homogéneos } \\
\hline Ecodriving & 5 & 5.2994 & X \\
\hline Normal & 5 & 7.66302 & $X$ \\
\hline
\end{tabular}

Método: 95,0 porcentaje LSD

Los resultados referentes al factor de emisión de CO se observa en la figura 6, donde la mayor emisión de CO se produce en la conducción normal. Debido a un mayor par motor determinado por un régimen mayor de revoluciones del motor el cual influye directamente en el coeficiente de aire (Gongbin y Chung, 2013); en el caso del ecodriving se trata de reducir y mantener constantes las revoluciones del motor; que influye directamente en el par motor medio, que se manifiesta en la reducción de CO debido a que se tiene un coeficiente de aire de modo casi li- 
neal con tendencia a mezcla rica. El monóxido de carbono se reduce porque se mantiene una velocidad media promedio mayor que la conducción normal (Haworth y Symmons, 2001), mientras que en Bishop, Stettler, Molden y Boises (2016) informan de que las emisiones de CO disminuyen monotónicamente con respecto a la velocidad instantánea, determinando que un auto que viaja a $120 \mathrm{~km} / \mathrm{h}$ emite $100 \%$ más de CO que al viajar a $88 \mathrm{~km} / \mathrm{h}$.

Figura 6. Medias comparativas del factor de emisión de CO según modo de conducción

Medias y $95,0 \%$ de Fisher LSD

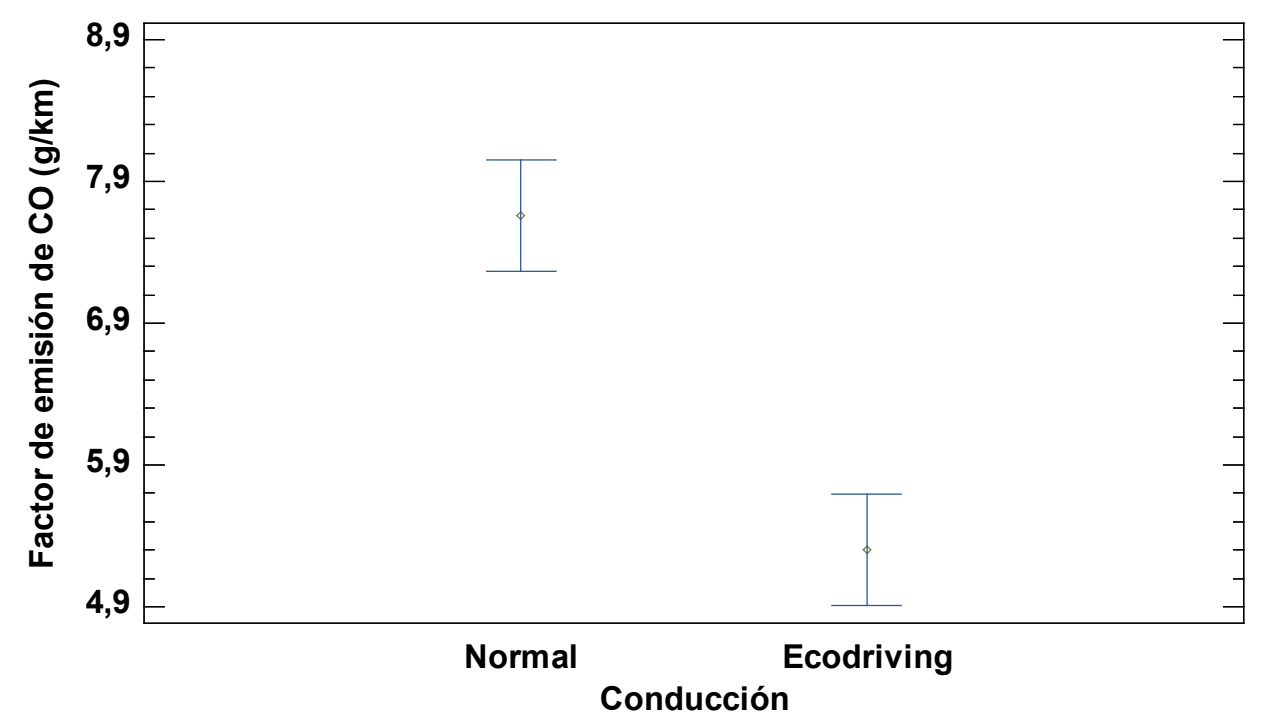

La tabla 6 indica que no existe diferencia significativa en las emisiones de HC. Como característica importante se estableció que la conducción ecodriving genera $0.016 \mathrm{~g} / \mathrm{km}$ más de emisión que al aplicar la conducción normal la cual no significa una diferencia significativa en la emisión y corresponde a un 11.59 \% de emisión. Lo cual difiere de los estudios obtenidos por Mensing, Bideaux, Trigui, Ribet y Jeanneret (2014) donde se obtiene una reducción de los HC del 7.4 \% en una simulación en un vehículo liviano a una altura de 169 msnm aplicando el ecodriving. La diferencia de dichos resultados se debe a que la altura influye directamente en la combustión, debido a que se reduce la cantidad de oxígeno en la mezcla, y bajo estas condiciones es menos eficiente, influyendo en las emisiones y el consumo de combustible (Zervas, 2011).

Tabla 6. Medias comparativas del factor de emisión de HC según modo de conducción

\begin{tabular}{|l|l|l|l|}
\hline & \multicolumn{1}{|c|}{ Casos } & \multicolumn{1}{|c|}{ Media } & Grupos Homogéneos \\
\hline Normal & 5 & 0.12156 & X \\
\hline Ecodriving & 5 & 0.138 & X \\
\hline
\end{tabular}

Método: 95.0 porcentaje LSD

En la figura 7, se observa que al conducir con ecodriving la emisión de hidrocarburos no combustionados aumenta de $0.1216 \mathrm{~g} / \mathrm{km}$ a $0.138 \mathrm{~g} / \mathrm{km}$, debido a que se reduce el par motor solicitado por el conductor lo que reduce la temperatura en la cámara de combustión y se incre- 
menta la zona de baja temperatura donde se forman los HC (Ramadhas, Singh, Sakthivel, Mathai y Sehgal, 2016). En relación con el estudio de Ho, Wong y Chang (2015) en el cual se obtiene una reducción de los HC del $10 \%$ a una altura de 15 msnm al aplicar una capacitación de 30 a 45 sesiones, se podría plantear la hipótesis si a esta altura después de realizar la misma capacitación se puede reducir las emisiones de $\mathrm{HC}$.

Figura 7. Comparativo del factor de emisión de HC

Medias y $95,0 \%$ de Fisher LSD

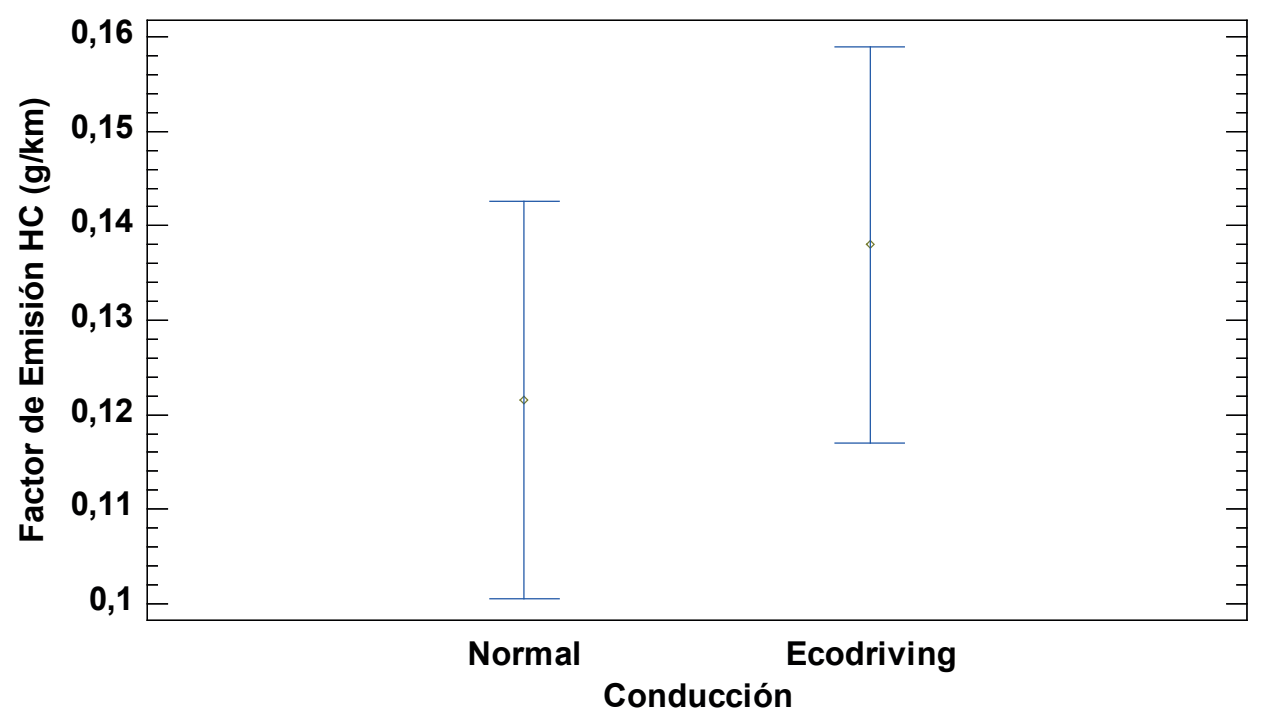

El valor promedio de la emisión de $\mathrm{NO}_{x}$ en los dos casos se muestra en la tabla 7, donde se refleja una reducción significativa de estas emisiones de $0.211 \mathrm{~g} / \mathrm{km}$, correspondiente al 20.38 \% del ecodriving, con respecto a la conducción normal. La tendencia a la reducción de estos gases se determina por la reducción del punto encendido debido a revoluciones más bajas y estables lo que conduce a menores presiones, menores temperaturas y una velocidad de combustión más lenta. Estos resultados se pueden corroborar con el estudio desarrollado por Kobayashi, Tsubota y Kawashima (2007) donde se determinó que al aplicar ecodriving se reduce la emisión en un $17.03 \%$ pero en $\mathrm{g} / \mathrm{h}$.

Tabla 7. Comparativas del factor de emisión del $\mathrm{NO}_{x}$

\begin{tabular}{|l|l|l|l|}
\hline & \multicolumn{1}{|c|}{ Casos } & \multicolumn{1}{c|}{ Media } & Grupos Homogéneos \\
\hline Ecodriving & 5 & 0.8272 & $X$ \\
\hline Normal & 5 & 1.0387 & $X$ \\
\hline
\end{tabular}

Método: 95.0 porcentaje LSD

Las emisiones de $\mathrm{NO}_{x}$ en la figura 8 muestran cambios significativos entre los dos tipos de conducción. Las emisiones varían en un 31.87 \% comparando entre el valor más alto de conducción normal y el más bajo del ecodriving, mientras que entre los valores más altos de 
los dos tipos de conducción hay una diferencia de $14.98 \%$ y con los menores valores hay una diferencia de $24.38 \%$ y la diferencia entre las medias es de $20.38 \%$, mediante la cual se indica que aplicando una técnica de conducción ecodriving con mayores horas de capacitación y con la ayuda de software se pueden alcanzar mayores valores de reducción, como se determinó en el estudio de Mensing, Bideaux, Trigui, Ribet y Jeanneret (2014) donde se obtuvo una reducción de $54.5 \%$ de NOx.

Figura 8. Comparativo del factor de emisión de $\mathrm{NO}_{x}$

Medias y $\mathbf{9 5 , 0} \%$ de Fisher LSD

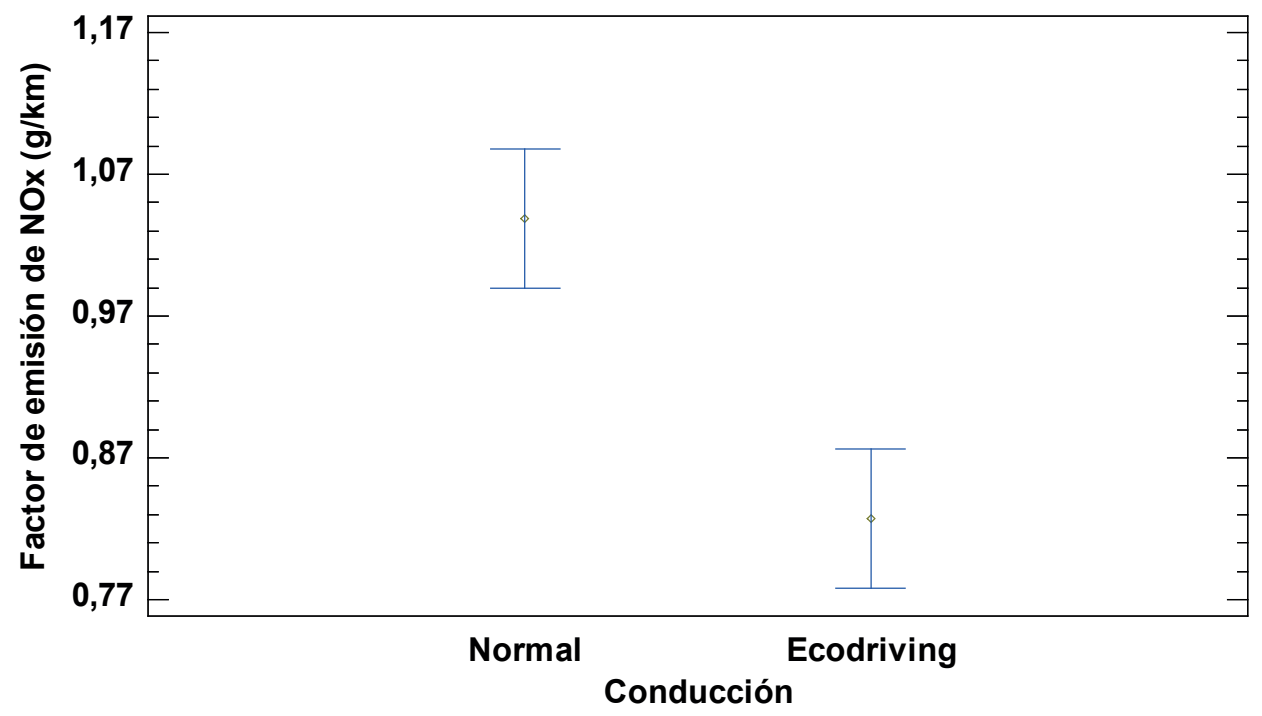

\section{Conclusiones y recomendaciones}

El análisis de consumo de combustible en el vehículo al aplicar el modo de conducción normal con respecto al ecodriving, evidenció la reducción del consumo de combustible y un aumento en el rendimiento de la distancia recorrida por cantidad de combustible consumido.

La prueba de ruta utilizando el equipo Onboard en el vehículo, permitió determinar los factores de emisión, mediante los cuales se concluye que se reducen significativamente las emisiones de gases $\mathrm{CO}$ y NOx, mientras que las emisiones de $\mathrm{HC}$ al aplicar ecodriving aumentan, pero no de manera significativa.

La implementación del ecodriving en un automóvil en la ciudad de Quito-Ecuador puede ser considerada como una estrategia para reducción de emisiones contaminantes y una herramienta para incrementar la eficiencia energética del combustible en un motor de combustión interna. 


\section{Bibliografía}

A, A. A., y Yasunori, M. (2013). The Effect of Ecodrive Program in Simulated and Real-World Driving Modes on the Fuel Economy of Manila Drivers. Journal of the Eastern Asia Society for Transportation Studies, 10: 1203-1217.

AEADE. (2018). ANUARIO 2017. Quito. Retrieved from www.aeade.net

Andrade-Castañeda, H. J., Arteaga-Céspedes, C. C. y Segura-Madrigal, M. A. (2017). Emisión de gases de efecto invernadero por uso de combustibles fósiles en Ibagué, Tolima (Colombia). Corpoica Ciencia y Tecnología Agropecuaria, 18 (1): 103-112. https://doi.org/10.21930/rcta.vol18_num1_ art:561

Andrieu, C., y Saint Pierre, G. (2014). Evaluation of ecodriving performances and teaching method: Comparing training and simple advice. European Journal of Transport and Infrastructure Research, 14 (3): 201-213.

Baric, D., Zovak, M. y Perisa, M. (2013). Effects of Eco-Drive Education on the Reduction of Fuel Consumption and CO2 Emissions. Promet- Traffic\&Transportation, 25 (3): 262-272.

Barth, M., y Boriboonsomsin, K. (2009). Energy and Emissions Impacts of a Freeway-based Dynamic Ecodriving System. Transportation Research Part D: Transport and Environment, 14 (6): 400-410.

Betella, A., y Verschure, P. F. M. J. (2016). The Affective Slider: A Digital Self-Assessment Scale for the Measurement of Human Emotions. PLOS ONE, 11 (2): e0148037. https://doi.org/10.1371/journal. pone.0148037

Beusen, B., BroekX, S., Denys, T., BeckX, C., Degraeuwe, B., Gijsbers, M. y Panis, L. I. (2009). Using on-board Logging Devices to Study the Longer-term Impact of an Eco-driving Course. Transportation Research Part D: Transport and Environment, 14 (7): 514-520.

Bishop, J., Stettler, M., Molden, N. y Boises, A. (2016). Engine maps of fuel use and emissions from transient driving cycles. Applied Energy, 183 (1): 202-2017. Retrieved from https://doi.org/10.1016/j. apenergy.2016.08.175

Bottiglione, F., Contursi, T., Gentile, A. y Mantriota, G. (2014). The fuel economy of hybrid buses: The role of ancillaries in real urban driving. Energies, 7 (7): 4202-4220.

Castillo, J. C., Restrepo, Á., Tibaquirá, J. E. y Quirama, L. F. (2019). Estrategias de eficiencia energética en vehículos livianos del transporte por carretera en Colombia. Revista UIS Ingenierías, 18 (3): 129 140. https://doi.org/10.18273/revuin.v18n3-2019013

Emberger, G. (2017). Low carbon transport strategy in Europe: A critical review. International Journal of Sustainable Transportation, 11 (1): 31-35. https://doi.org/10.1080/15568318.2015.1106246

Energy Information Administration. (2017). International Energy Outlook. Retrieved from https://www.eia. gov/outlooks/ieo/pdf/0484(2017).pdf

Fernández-Rodríguez, A., Fernández-Cardador, A. y Cucala, A. P. (2015). Energy efficiency in high speed railway traffic operation: A real-time ecodriving algorithm. 2015 IEEE 15th International Conference on Environment and Electrical Engineering, EEEIC 2015-Conference Proceedings. https:// doi.org/10.1109/EEEIC.2015.7165181

Guardia, Y., Márquez, |J., Sánchez, V., Llanes, E., Rocha, J. y Peralta, D. (2018). Enhancements to the subject Statistical Design of Experiments for students of the Mechanical Engineering careere. Espacios, 39 (30): 1-16. Retrieved from https://www.revistaespacios.com/a18v39n30/18393010.html

Ho, S., Wong, Y. y Chang, V. (2015). What Can Eco-driving Do for Sustainable Road Transport? Perspectives from a City (Singapore) Eco-driving Programme. Sustainable Cities and Society, 14: 82-88.

Ho, S. H., Wong, Y. D. y Chang, V. W. C. (2015). What Can Eco-driving Do for Sustainable Road Transport? Perspectives from a City (Singapore) Eco-driving Programme. Sustainable Cities and Society, 14: 82-88.

Kobayashi, I., Tsubota, Y. y Kawashima, H. (2007). Eco-driving simulation: Evaluation of eco-driving within a network using traffic simulation. In URBAN TRANSPORT. Retrieved from 10.2495/UT070701 
Larue, G., Malik, H., Rakotonirainy, A. y Demmel, S. (2014). Fuel consumption and gas emissions of an automatic transmission vehicle following simple eco-driving instructions on urban roads. IET Intelligent Transport Systems, 8 (7): 590-597. https://doi.org/10.1049/iet-its.2013.0076

Leguisamo, J., Celi, S., Noroña, M. y Puente, E. (2018). Estudio de Emisiones Contaminantes Producidas por un Motor MEP Con Transmisión Automática y Transmisión Manual. INNOVA Research Journal, 3 (4): 120-128.

Leguisamo, J., Llanes, E., Noroña, M. y Puente, E. (2018). Identificación del modo de trabajo de un motor GDI al variar la altura mediante una prueba estática a ralentí y 2500 Rpm. INNOVA Research Journal, 3 (7): 61-70.

Llanes Cedeño, E. A., Rocha-Hoyos, J. C., Peralta Zurita, D. B. y Leguísamo Milla, J. C. (2018). Evaluación de emisiones de gases en un vehículo liviano a gasolina en condiciones de altura. Caso de estudio Quito, Ecuador. Enfoque UTE, 9 (2): 149-158. https://doi.org/10.29019/enfoqueute.v9n2.201

Magaña, V. C. (2014). Eco-driving: ahorro de energía basado en el comportamiento del conductor. Madrid: Universidad Carlos III de Madrid. Retrieved from http://e-archivo.uc3m.es/handle/10016/19981

Mensing, F., Bideaux, E., Trigui, R., Ribet, J. y Jeanneret, B. (2014). Eco-driving: An Economic or Ecologic Driving Style? Transportation Research Part C: Emerging Technologies 38: 110-121.

Morello, E., Toffolo, S. y Magra, G. (2016). Impact Analysis of Ecodriving Behaviour Using Suitable Simulation Platform (ICT-EMISSIONS Project). In Transportation Research Procedia. https://doi. org/10.1016/j.trpro.2016.05.252

Movilidad, S. de. (2014). Diagnóstico de la movilidad en el Distrito Metropolitano de Quito para el plan metropolitano de desarrollo territorial. Quito.

N. Haworth y M. Symmons. (2001). The relationship between fuel economy and safety outcomes. Monash University Accident Research Centre.

Orfila, O., Freitas Salgueiredo, C., Saint Pierre, G., Sun, H., Li, Y., Gruyer, D. y Glaser, S. (2017). Fast computing and approximate fuel consumption modeling for Internal Combustion Engine passenger cars. Transportation Research Part D: Transport and Environment, 50: 14-25. https://doi. org/10.1016/j.trd.2016.10.016

Orfila, Olivier, Saint Pierre, G. y Andrieu, C. (2012). Gear Shifting Behavior Model for Ecodriving Simulations Based on Experimental Data. Procedia-Social and Behavioral Sciences, 54: 341-348. https:// doi.org/10.1016/j.sbspro.2012.09.753

Quichimbla, F. y Solís, J. (2017). Desarrollo de ciclos de conducción en ciudad, carretera y combinado para evaluar el rendimiento real del combustible de un vehículo con motor ciclo Otto en el Distrito Metropolitano de Quito. Escuela Politécnica Nacional.

Ramadhas, A. S., Singh, P. K., Sakthivel, P., Mathai, R. y Sehgal, A. K. (2016). Effect of Ethanol-Gasoline Blends on Combustion and Emissions of a Passenger Car Engine at Part Load Operations. SAE Technical Paper. https://doi.org/10.4271/2016-28-0152

Rionda, Al. Martínez, D., Pañeda, X., Arbesú, D. y Jiménez, E. (2012). Sistema Tutor Para La Conducción Eficiente de Vehículos de combustion. IEEE-RITA, 7 (3): 133-140.

Rocha-Hoyos, J. C., Tipanluisa, L. E., Zambrano, V. D. y Portilla, Á. A. (2018). Estudio de un Motor a Gasolina en Condiciones de Altura con Mezclas de Aditivo Orgánico en el Combustible. Información Tecnológica, 29 (5): 325-334. https://doi.org/10.4067/S0718-07642018000500325

Rodríguez, R. A., Virguez, E. A., Rodríguez, P. A. y Behrentz, E. (2016). Influence of driving patterns on vehicle emissions: A case study for Latin American cities. Transportation Research Part D: Transport and Environment, 43: 192-206. https://doi.org/10.1016/j.trd.2015.12.008

Rolim, C., Baptista, P., Duarte, G., Farias, T., and Shiftan, Y. (2014). Quantification of the Impacts of Ecodriving Training and Real-time Feedback on Urban Buses Driver's Behaviour. Transportation Research Procedia, 3: 70-79. 
Rutty, M., Matthews, L., Andrey, J. y Del Matto, T. (2013). Eco-driver Training within the City of Calgary's Municipal Fleet: Monitoring the Impact. Transportation Research Part D: Transport and Environment, 24: 44-51.

Secretaría de Ambiente. (2017). Informe Calidad del aire -2016 distrito Metropolitano de Quito. Quito. Retrieved from http://www.quitoambiente.gob.ec/ambiente/index.php/informes\#informe-calidad-del-aire-2016

Stillwater, T., Kurani, K. S. y Mokhtarian, P. L. (2017). The combined effects of driver attitudes and in-vehicle feedback on fuel economy. Transportation Research Part D: Transport and Environment, 52: 277-288. https://doi.org/10.1016/j.trd.2017.02.013

Yanzhi, X. y Hanyan, Li., Haobing, Liu., Rodgers, M. y Guensler, R. (2017). Eco-driving for transit: An effective strategy to conserve fuel and emissions. Applied Energy, 194: 784-797. https://doi.org/http:// dx.doi.org/10.1016/j.apenergy.2016.09.101

Zervas, E. (2011). Impact of altitude on fuel consumption of a gasoline passenger car. Fuel, 90 (6): 23402342. https://doi.org/10.1016/j.fuel.2011.02.004 